\title{
A comment on "What catch data can tell us about the status of global fisheries" (Froese et al. 2012)
}

\author{
R. M. Cook
}

Received: 14 November 2012/ Accepted: 24 January 2013

(C) Springer-Verlag Berlin Heidelberg 2013

There is considerable interest in the state of the world's natural fishery resources. The paper by Froese et al. (2012) is a recent example of applying a set of ad hoc decision rules to a time series of catch data in order to assign the world's fisheries to categories of exploitation and hence make generalisations about their current status. They conclude that the percentage of stocks that are over-exploited is worse than previously reported in FAO (2010). The approach used by Froese et al. is based on an algorithm proposed by Froese and Kesner-Reyes (2002) which has been heavily criticised both on theoretical grounds and from simulation studies (Branch et al. 2011; Daan et al. 2011; Wilberg and Miller 2007). In their recent paper, Froese et al. (2012) produce additional analyses to support their method which assumes that maximum sustainable yield (MSY) lies in the interval $\left(0.5 C_{\max }, C_{\max }\right)$, where $C_{\max }$ is the maximum observed catch in the time series. Unfortunately, these analyses do not support their contention that MSY for a particular stock is related to maximum catch in a predictable way and renders their conclusions unsafe.

Froese et al. suggest that $C_{\max }$ is highly correlated with MSY for a set of fully assessed fish stocks in the northeast Atlantic. Whilst the coefficient of determination, $R^{2}$, appears impressively high (Fig. 1a), it hides the fact that the relationship, which is plotted on a log scale, is simply a

Communicated by M. Coll.

R. M. Cook ( $\square)$

MASTS Marine Population Modelling Group,

Department of Mathematics and Statistics,

University of Strathclyde, Livingstone Tower,

26 Richmond Street, Glasgow G1 1XH, Scotland, UK

e-mail: robin.cook@strath.ac.uk feature of the fact that the stocks examined differ by several orders of magnitude. The variation in catch within a stock is far less than the variation in catch between stocks, so choosing two values from each stock $\left(C_{\max }, \mathrm{MSY}\right)$ results in taking two values of very similar magnitude. Hence, plotting these pairs where each stock has a vastly different scale is little different from plotting $x$ on $x$. It is obvious that small stocks will have a low MSY and large stocks will have a high MSY. It does not mean $C_{\max }$ can predict MSY, or vice versa, with any useful precision.

It can be shown through a simple simulation that the correlation is largely unrelated to a relationship between MSY and $C_{\max }$. In the simulation, a catch is selected at random from each stock and the $R^{2}$ calculated for each realisation of the relationship between log catch and log MSY. The result of a series of 10,000 simulations is shown in Fig. 1. Figure 1a shows the original relationship reported by Froese et al. Also shown is the relationship that had the highest $R^{2}$ based on selecting a catch at random. As can be seen, it is possible to obtain a better $R^{2}$ using just a random catch. While obtaining an $R^{2}$ value larger than the $C_{\max }$ relationship is fairly rare in the simulations, it is not fortuitous. Figure $1 \mathrm{~b}$ shows the frequency distribution of the $R^{2}$ values from the simulations. Nearly all the $R^{2}$ values are larger than 0.9 with the mode at 0.95 . In effect, any random catch from the time series is highly correlated with MSY when examined across stocks of widely differing magnitude. Hence, the statement by Froese et al. that MSY explains $98 \%$ of the variance in $C_{\max }$ hides the fact that almost all of the variation explained is due to the difference in scale between stocks since MSY will explain most of the variation in any randomly chosen catch.

The high correlation between $C_{\max }$ and MSY fails when the effect of stock size is accounted for. Froese et al. give the regression equation for the relationship in Fig. 1a as: 
Fig. 1 a The relationship between log maximum catch and $\log$ MSY for 50 assessed stocks (open circles) as reported by Froese et al. (2012). Also shown is the relationship with the highest $R^{2}$ between the $\log$ of a randomly chosen catch from each stock and the log of the respective MSY (solid circles). b The frequency distribution of $R^{2}$ from 10,000 simulations of selecting catches at random and correlating the $\log$ of these with $\log$ MSY. c The relationship between log maximum catch and log MSY when the catch series and MSY are normalised to their respective means. d The frequency distribution of the ratio $\mathrm{MSY} / C_{\max }$ for 50 assessed stocks. The heavy horizontal line shows the $95 \%$ confidence interval based on a lognormal distribution. Data are taken from Froese and Proelß (2010) (a)

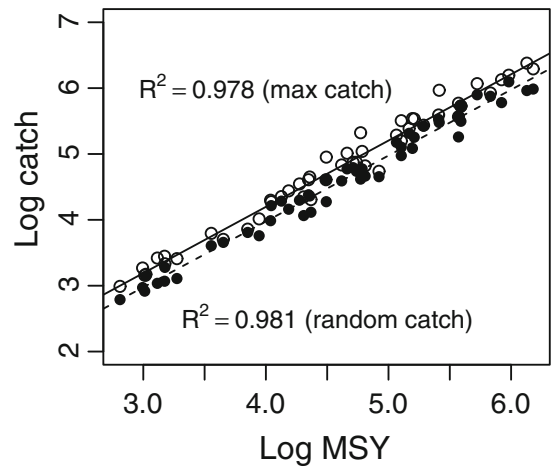

(b)

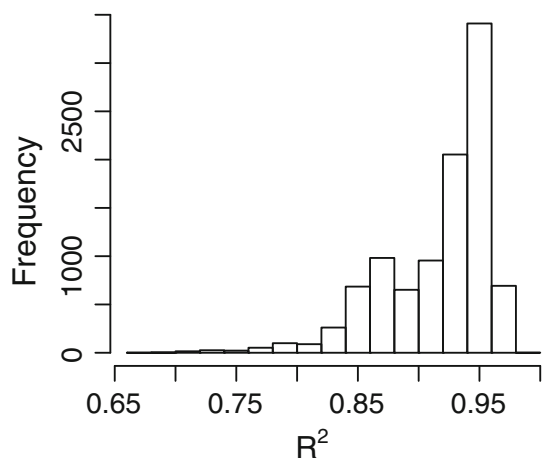

(c)

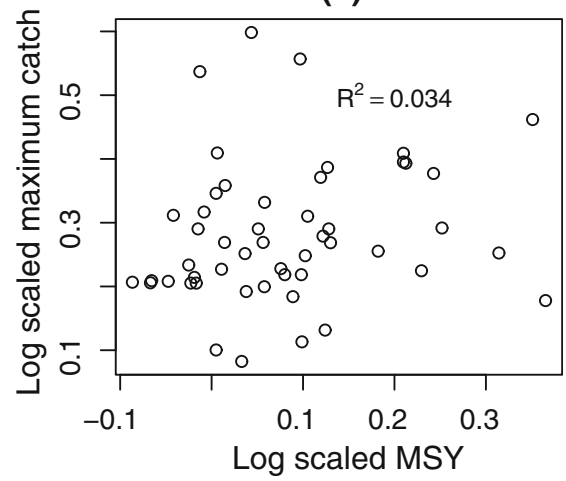

(d)

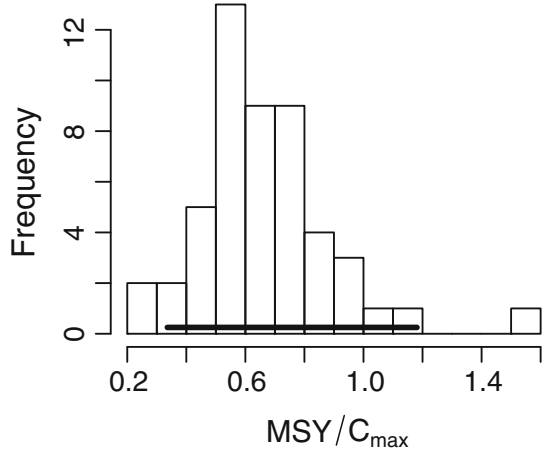

$\log _{10}\left(C_{\max }\right)=1.00036 \log _{10}(\mathrm{MSY})+0.1822$

Since the slope is effectively 1 , this means there is a proportional relationship between $C_{\max }$ and MSY with a constant ratio, $r$, of $10^{0.1822}$, that is, $C_{\max } / \mathrm{MSY}=r$. If this ratio really is constant, then it should hold for any arbitrary change in scale of $C_{\max }$ and MSY since:

$\frac{C_{\max }}{\mathrm{MSY}}=\frac{b C_{\max }}{b \mathrm{MSY}}$, where $b$ is an arbitrary scaling constant.

Figure 1c shows $C_{\max }$ plotted against MSY when each stock has been scaled to its series mean (i.e. each catch series been normalised to a mean of 1). With this correction to account for scale, the correlation all but disappears. MSY now only explains $3.4 \%$ of the variation in $C_{\max }$ showing that there is no relationship between $C_{\max }$ and MSY.

Froese et al. also state that the $95 \%$ confidence interval for the median value of $\mathrm{MSY} / C_{\max }$ (i.e. $\left.1 / r\right)$ is $(0.56,0.7)$. The implication of this statistic is that the ratio does not vary much, and hence, the assumption that MSY lies in the interval $\left(0.5 C_{\max }, C_{\max }\right)$ is justified. However, all this interval says is that the mid-point of all the $1 / r$ values does not vary much when examined across stocks. In fact what is of interest is the overall variation of $1 / r$ across stocks as we wish to know the likely ratio when we apply the decision algorithm to a new stock. Figure 1d shows the frequency distribution of MSY/ $C_{\max }$ with the sample $95 \%$ confidence interval based on a lognormal distribution. It shows that the ratio is highly variable lying in the interval $(0.34,1.19)$ and does not support the assumption of an interval of $(0.5,1)$. It means that in assuming a narrower interval, the decision rule will be prone to misclassification.

Perhaps one way to improve the classification algorithm would be to use the frequencies in Fig. 1d to assign a probability distribution to the exploitation status of the stock, rather than using ad hoc knife-edge criteria. It would avoid the need to make strong assumptions about the size of MSY in relation to $C_{\max }$. This still suffers from the problem of linking catch size to exploitation rate without making further assumptions about the development of the fishery, however. Alternatively, one could derive a probability distribution for exploitation status associated with each catch ratio $\left(C / C_{\max }\right)$ based on stocks with full assessment data. The advantage of such an approach is that it explicitly considers measured harvest rates rather than simply judging catches in relation to MSY.

It is well known that catch data alone do not contain sufficient information about exploitation rates to estimate stock status because the observed catch is the product of both exploitation rate and stock size. For example, small catches may be explained either by a low exploitation rate with a large stock or a high exploitation rate with a small stock. Froese et al. seek to circumvent this problem by 
making strong assumptions about the catch series and their respective fisheries. Unfortunately, these assumptions are not yet supported by a sound analysis and it means that their conclusions on the status of the world's fish stocks are not reliable.

\section{References}

Branch TA, Jensen OP, Ricard D, Ye Y, Hilborn R (2011) Contrasting global trends in marine fishery status obtained from catches and from stock assessments. Conserv Biol. doi: 10.1111/j.1523-1739.2011.01687.x
Daan N, Gislason H, Pope JG, Rice JC (2011) Apocalypse in world fisheries? The reports of their death are greatly exaggerated. ICES J Marine Sci. doi:10.1093/icesjms/fsr069

FAO (2010) The state of world fisheries and aquaculture 2010. FAO, Rome

Froese R, Kesner-Reyes K (2002) Impact of fishing on the abundance of marine species. ICES Document CM 2002/L:12, p 15

Froese R, ProelB A (2010) Rebuilding fish stocks no later than 2015: will Europe meet the deadline? Fish Fish 11:194-202

Froese R, Zeller D, Kleisner K, Pauly D (2012) What catch data can tell us about the status of global fisheries. Mar Biol 159: 1283-1292. doi:10.1007/s00227-012-1909-6

Wilberg MJ, Miller TJ (2007) Comment on "impacts of biodiversity loss on ocean ecosystem services', Science 316:1285 